\title{
Asymmetric Supernova Explosion Investigated by Geodetic Precession
}

\author{
M. Kramer ${ }^{1}$, N. Wex ${ }^{2}$, V. Kalogera ${ }^{3}$, A. Karastergiou ${ }^{2}$ \\ ${ }^{1}$ Jodrell Bank Observatory, University of Manchester, UK \\ ${ }^{2}$ Max-Planck-Institut für Radioastronomie, Bonn, Germany \\ ${ }^{3}$ Harvard-Smithsonian Center for Astrophysics, Cambridge, USA
}

\begin{abstract}
We use observations and modelling of geodetic precession in the PSR B1913+16 system to obtain direct constraints on direction and magnitude of kicks imparted to the pulsar companion during the most recent supernova explosion. We find that the kick must have been directed almost perpendicular to the rotation axis of the progenitor star.
\end{abstract}

\section{Geodetic precession}

The effects of geodetic precession, a relativistic spin-orbit coupling, have been identified in the double neutron star systems PSR B1913+16 (Weisberg et al. 1989, Kramer 1998) and PSR B1534+12 (Stairs et al. 2000). Geodetic precession is predicted by general relativity for cases when the pulsar spin and the orbital momentum vector are misaligned. As the pulsar spin precesses around the total angular momentum vector, the pulsar's orientation towards Earth changes slowly with time. The different cuts of our line-of-sight through the pulsar emission beam result in secular changes of the pulse profiles.

For PSR B1913+16, Kramer (1998) was able to detect an expected decrease in component separation of the double pulse profile. Assuming a circular hollowcone-like beam and a precession rate of $\Omega=1.21 \mathrm{deg} / \mathrm{yr}$ as predicted by general relativity, Kramer was able to model the complete geometry of the binary system. The misalignment angle between orbital angular momentum and pulsar spin was determined to $\lambda=22_{-8}^{+4} \mathrm{deg}$. Recently, Weisberg \& Taylor (2000) confirmed these measurement, and Karastergiou et al. (2000) presented a method to include polarization measurements in the modelling process.

\section{Asymmetric Supernovae \& Kicks}

Geodetic precession observed in double neutron stars is direct evidence for asymmetries occurring in supernova explosions: Both PSRs B1534+12 and B1913+16 are recycled pulsars which have accreted mass and angular momentum from their companion before these exploded in supernova (SN) events. Due to the angular momentum transfer and a following common envelope phase, a corresponding pre-SN system is expected to consist of a He-star and a recycled pulsar in a circular orbit, where all spin vectors (i.e. orbital angular momentum, pulsar spin and companion spin) are aligned. An asymmetry in the explosion of the He-star leads to a kick imparted to the newly born neutron star. Unless the kick is directed 

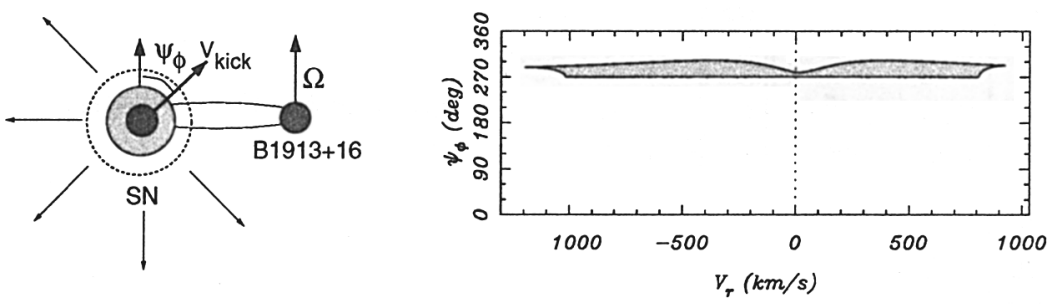

Figure 1. Polar angle of the kick as a function of the unknown radial velocity for PSR B1913+16 in a pro-grade case. For details see Wex et al. (2000). All cases lead to the same conclusion that the kick was perpendicular to the rotation axis of the exploding star.

favorably, the binary system disrupts (Fryer \& Kalogera 1997). The survival of systems with characteristics as those of PSRs B1913+16 and B1534+12 requires kick magnitudes of $\gtrsim 300 \mathrm{~km} \mathrm{~s}^{-1}$ and $\gtrsim 200 \mathrm{~km} \mathrm{~s}^{-1}$, respectively.

\section{Constraints on Kick Mechanisms}

Since the recycled pulsar is unaffected by the explosion of its companion, its spin vector will continue to point in the direction of the pre-SN orbital angular momentum. Its misalignment with the post-SN orbital spin can be measured by geodetic precession, yielding the relative orientation of the pre- and post-SN orbits. Because of their compact nature, the neutron stars do not interact, but the system's evolution is solely determined by the emission of gravitational waves and its motion in the galactic potential. Computations back in time provide the orbit configuration just after the second SN (Wex et al. 2000). Combining all information and constraints (e.g. conservation of momentum etc.), those kick parameters can be derived which are allowed to explain the observed configuration. With the unmeasurable radial velocity as the only free parameter and considering different cases, Wex et al. reached a number of interesting conclusions for PSR B1913+16. These include: a) retrograde solutions typically require larger kicks, b) kick velocities between $300 \mathrm{~km} \mathrm{~s}^{-1}$ and $1100 \mathrm{~km} \mathrm{~s}^{-1}$ are necessary, c) the kick must have been almost perpendicular to the spin axis of the progenitor He-star, d) the kick must have been short compared to the birth spin period of the neutron star and d) in case of an alignment of the progenitor's magnetic and spin axes, the kick cannot have been in direction of the magnetic field.

\section{References}

Fryer, C., \& Kalogera, V., 1997, ApJ, 489, 244

Karastergiou, A., et al., 2000, PASP, 202, 125

Kramer, M. 1998, ApJ, 509, 856

Stairs ,I. H., et al., 2000, PASP, 202, 121

Weisberg, J.M., Romani, R., \& Taylor, J.H. 1989, ApJ, 347, 1029

Weisberg, J.M., \& Taylor, J.H. 2000, PASP, 202, 127

Wex, N,, Kalogera, V., \& Kramer, M., 2000, ApJ, 528, 401 